\title{
Multigap superconductivity and barrier-driven resonances in superconducting nanofilms with an inner potential barrier
}

\author{
Mauro M. Doria,,${ }^{1,2, *}$ Marco Cariglia,,$^{1,3, \dagger}$ and Andrea Perali ${ }^{1,4, \ddagger}$ \\ ${ }^{1}$ School of Pharmacy, Physics Unit, Università di Camerino, 62032 - Camerino, Italy \\ ${ }^{2}$ Instituto de Física, Universidade Federal do Rio de Janeiro, 21941-972 Rio de Janeiro, Brazil \\ ${ }^{3}$ Departamento de Física, Universidade Federal de Ouro Preto, 35400-000 Ouro Preto Minas Gerais, Brazil \\ ${ }^{4}$ INFN, Sezione di Perugia, 06123 - Perugia, Italy
}

(Received 19 June 2016; revised manuscript received 16 November 2016; published 20 December 2016)

\begin{abstract}
We study the crossover in a zero-temperature superconducting nanofilm from a single to a double superconducting slab induced by a barrier in the middle. We use the Bogoliubov-de Gennes (BdG) equations in the Anderson approximation to show that the single-phase superconducting ground state of this heterostructure is intrinsically multigapped and has a new type of resonance caused by the strength of the barrier, thus distinct from the Thompson-Blatt shape resonance which is caused by tuning the thickness of the film. The simplest theoretical framework able to describe a finite height and very thin tunable insulating potential barrier in the middle is provided by a $\delta$-function potential. In this framework, the even single-particle states are affected by the insulating barrier, whereas the odd ones are not. The new type of resonance, hereafter called barrier-driven resonance, is caused by the crossing of the even single-particle states through the Fermi surface. The lift of the even-odd degeneracy at the barrier reconfigures the pairing interaction and leads to a multigapped superconducting state with barrier-driven resonances.
\end{abstract}

DOI: 10.1103/PhysRevB.94.224513

\section{INTRODUCTION}

Since two-gap superconductivity has been discovered in $\mathrm{MgB}_{2}$ [1-7] an intense research activity has been devoted to multiband and multigap superconductors. In fact twogap superconductivity has been theoretically proposed long ago $[8,9]$ and recently found in many compounds [10] ranging from composites [11] to metallic $\mathrm{Pb}[12,13]$. Nanoengineered superconducting films tailored to atomic precision thickness [14-16] are pivotal to this research because they display multibands and multigapped superconductivity. Superconductivity in ultrathin nanofilms was demonstrated to be very robust and can survive even in monatomic layers of In and $\mathrm{Pb}[16]$.

In this paper we show that a superconducting nanofilm develops new properties by the addition of a potential barrier in its center. These properties are an intrinsic multigapped structure and a new type of resonance driven by the potential barrier. It is well known that a superconducting nanofilm (without the potential barrier) displays a multigapped structure, but only within the narrow window of shape resonances, as shown by Thompson and Blatt [17]. However, this narrow window, defined by the nanofilm width and by the pairing energy scale, renders very difficult its experimental observation. The potential barrier turns the superconducting film into a SIS nanofilm: a superconductor-insulator-superconductor "sandwich." The two superconductors are thin slabs in the quantum-size regime separated by a very thin insulating slab, represented by the potential barrier itself. We show here that the SIS nanofilm is multigapped because it remains as such outside the shape-resonance windows and possesses a new

\footnotetext{
*mauromdoria@gmail.com

†marco@iceb.ufop.br

${ }^{\ddagger}$ Andrea.Perali@unicam.it
}

type of resonance hereafter called barrier-driven resonance, which is distinct from the well-known shape-driven resonance occurring in single-slab superconducting nanofilms. Thus the SIS nanofilm displays very distinct and novel properties with respect to the single-slab nanofilm which stem from the additional parity properties brought by the insulating barrier put in its center. Single-particle states can be either even or odd with respect to it. Even states strongly feel the presence of the barrier while odd ones do not and this causes an additional splitting of the single-particle energy levels which is at the heart of the multigapped structure of the superconducting state.

The well-known shape-driven resonances predicted by Thompson and Blatt [17] more than fifty years ago and the present barrier-driven resonances proposed here share a single common origin albeit their distinct properties. To understand these common features, recall that single-particle electronic states in films are a sum over continuum and discrete degrees of freedom, associated with the parallel and perpendicular to the film surface degrees of freedom, respectively. The origin of the discreteness is in the quantum size regime that renders the superconducting gap smaller than the splitting between two consecutive energy levels perpendicular to the nanofilm. The discreteness no longer holds for a thick film since consecutive single-particle states become so close in energy that their splitting is smaller than the superconducting gap. The continuum treatment is always suited for the degrees of freedom parallel to the nanofilm surface, where the separation between consecutive levels is always smaller than the superconducting gap.

Superconducting nanofilms have a far richer structure than a bulk superconductor, the latter defined here by the simplest possible model, i.e., that of a single spherically symmetric three-dimensional Fermi surface. Even within this simple description, nanofilms are far more complex than the bulk. They display multiple two-dimensional Fermi surfaces, each associated with a distinct discrete state induced by the perpen- 
dicular confinement. Shape and barrier-driven resonances are a consequence of these multiple Fermi surfaces in nanofilms that cause a reconfiguration of the pairing interaction. The intersection of the chemical potential with the parabolic parallel bands defines multiple Fermi surfaces, where an attractive interaction leads to superconducting gaps around the Debye energy window centered in the chemical potential itself. A resonance in the superconducting gap is the consequence of the entrance (exit) of a two-dimensional Fermi surface into the Debye energy window. An intersection with a two-dimensional Fermi surface can be either added or removed and this affects the superconducting gap of the nanofilm. This phenomenon has no counterpart in the simplest description of the bulk superconductor. The adjustment of some nanofilm parameters moves the discrete levels and is responsible for resonances. The shape-driven resonance [18,19] is triggered by a change in the nanofilm thickness that affects the discrete levels. This is the only way to make a single-slab superconducting nanofilm resonate because it lacks any other internal structure. However, the SIS nanofilm has an internal structure given by the insulating barrier, and this opens a new venue for resonances through the adjustment of the discrete levels by the barrier strength. As shown here the even discrete levels are very sensitive to the barrier strength and through this mechanism can be moved to enter the Debye energy window and cause a resonance in the superconducting gap.

To unveil the intrinsic multigap structure and the existence of barrier-driven resonances in the SIS nanofilm we study its zero-temperature properties under a fixed chemical potential. Indeed the chemical potential can be considered approximately constant in case the number of atomic monolayers in the superconducting nanofilm surpasses a small number, known to be nearly five according to Fig. 1 of Ref. [17]. Such assumptions add simplicity to our study without compromising the present goals.

In this paper we consider that the SIS nanofilm has only one single superconducting state in thermodynamical equilibrium, which means that there is no Josephson current between the left and right sides of the potential barrier. The Josephson current exists in the case of a phase difference between two superconducting states separated by the potential barrier [20,21]. Here, Cooper pairs tunnel through the insulating barrier and there is no phase difference between these two sides. Although we compute here excited states (multigaps) of the SIS nanofilm those are assumed to be translational invariant along the film surface and so there is no spontaneous current passing from one side to the other of the SIS nanofilm. Thus Josephson vortices are excluded because those induce localized circulating currents from one slab to the other.

We use the Bogoliubov-de Gennes (BdG) equations in the Anderson approximation to show that superconductivity is multigapped and also to show the existence of barrier-driven resonances in the SIS nanofilm. We do it in the simplest possible theoretical framework where the finite insulating barrier is described by a repulsive $\delta$-function potential which retains the following two properties: The single-particle electronic states are either affected (even) or not (odd) by the barrier. The even states are only affected if their wave number falls below a threshold which describes the height of the finite barrier. Therefore we stress here that the $\delta$-function approach is able to describe a finite potential barrier. We find the present simple approach useful with respect to more elaborate treatments $[22,23]$ that can deal with features such as the proximity effect in the SIS nanofilm.

Thus our results are consistent with those found in Ref. [24], which shows that, for reduced dimensionality, the effect of impurities is significant and cannot be ignored. Indeed, the $\delta$-function barrier in the SIS nanofilm can be regarded as a translational invariant line of impurities cast in the center. Consequently, the symmetries of the superconducting subband gaps are affected, resulting in a multigapped homogeneous state only along the surface of the SIS nanofilm but not orthogonal to it, where the state has been known to be inhomogeneous [25].

The importance of the boundary conditions must be emphasized in the description of nanofilms through the $\mathrm{BdG}$ equations [26]. Here we take the standard Thompson-Blatt boundary conditions to show that the SIS nanofilm possesses barrier-driven resonances and an intrinsic multigap structure whose effects on the critical temperature will be considered elsewhere. A recent detailed investigation of the shape resonances at the critical temperature for a single superconducting nanofilm has been reported in Refs. [27,28] by an exact numerical solution of the BCS mean-field equations at fixed density. The main outcome is that the precise form of the shape resonance and the enhancement (or suppression) of the critical temperature depend on the strength of the confining potential at the nanofilm surface and on the value of the pairing interaction.

The enhancement of superconductivity in a shaperesonance scenario has been proposed and measured; namely, that obtained by shrinking all the dimensions of a superconductor to the nanoscale, as in nanoparticles, nanoclusters, and nanocubes [29-32]. In addition, there has been the proposal of shape resonances in superconducting nanofilms of $\mathrm{Pb}[14,33,34]$.

From the experimental point of view there are several ways to realize the insulating barrier of the SIS nanofilms. One possibility is to deposit on the $\mathrm{Nb}$ nanofilm a thin film of $\mathrm{Al}$ and then proceed with the in situ oxidation of $\mathrm{Al}$ at room temperature. The thickness of the $\mathrm{AlO}_{x}$ insulating barrier will change depending on the oxygen exposure time, allowing for a control of the barrier-potential strength [35]. $\mathrm{AlO}_{x}$ barriers with thickness of the order of $1 \mathrm{~nm}$ should be realizable. Another possibility could be to use silicon as an insulating barrier in the SIS nanofilms.

The paper is organized as follows: In Sec. II we introduce the physical parameters of the three metals, aluminum, lead, and niobium, which are used here to discuss the SIS nanofilm. Nevertheless, niobium is chosen to obtain physical values for the gaps. Notice that the SIS nanofilm has two distinct but equivalent limits of no barrier and of an extremely strong barrier. In these two limits the BdG equations should describe the same physics which indeed it does, as shown here. The multiband and multigap properties in these two limits are described in Sec. III. In particular, we show that, for an extremely strong barrier, the present formalism no longer holds because even and odd levels are so close to each other that cross pairing must be considered. The general formalism and equations to obtain the gaps for the even states, which are those that feel the barrier, are developed here. In 
Sec. IV we describe in detail the multigap structure close to a shape resonance. Section $\mathrm{V}$ is dedicated to the barrier-driven resonance proposed herein. We discuss our results in the context of other contributions to this field in Sec. VI and finally reach conclusions in Sec. VII. Appendix A summarizes the Anderson approximation for the Bogoliubov-de Gennes equations applied to a superconducting thin film, while the Schrödinger equation is analyzed in Appendix B, where the analytical expression of the interaction matrix can be found.

\section{DESCRIPTION OF INSULATING SLAB}

The potential barrier of the SIS nanofilm is modeled by a $\delta$ function [36],

$$
V_{b}(x)=\frac{\hbar^{2}}{m} \Omega \delta(x)=V_{0} \delta\left(\frac{x}{2 \varepsilon}\right),
$$

and the parameter $\Omega$ has the physical meaning of a wave number that sets the height of the barrier. Single-particle states with wave numbers much higher than $\Omega$ do not feel it. Thus the $\delta$ function, which diverges at the origin, is able to describe the physical properties of a very thin finite-height barrier. The thickness $2 a$ and $2 \varepsilon$ are of the SIS nanofilm and of the potential barrier, respectively, as shown in Fig. 1. The potential height $V_{0}$ describes the very thin insulating slab $2 \varepsilon$ sandwiched between two superconducting nanofilms of thickness $a-\varepsilon$. The particles are confined inside the SIS nanofilm since at the edges $-a$ and $a$ there is an infinite barrier, such as considered by Thompson and Blatt [17]. Figure 2

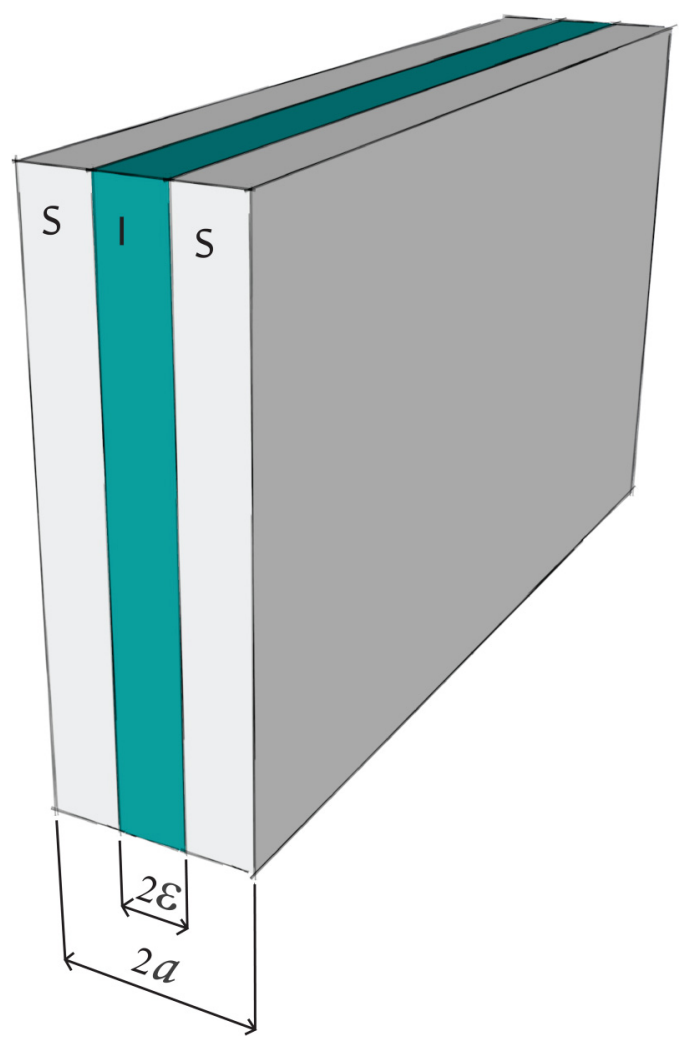

FIG. 1. A pictorial view of the SIS nanofilm composed of two superconducting slabs with thickness $a-\varepsilon$ and an insulating barrier with thickness $2 \varepsilon$ such that the total thickness is $2 a$.

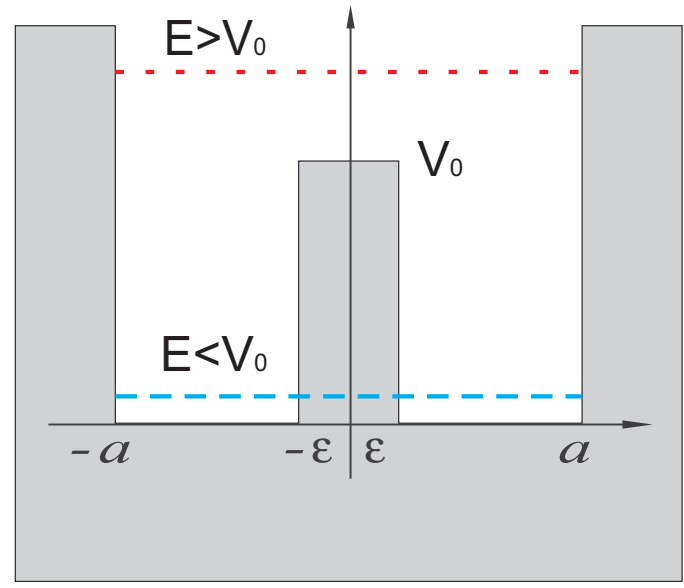

FIG. 2. Profile of the insulating barrier in the middle of the superconducting nanofilm. Single-particle energy levels above or below the barrier (height $V_{0}$ ) are shown here.

depicts the rectangular barrier of height $V_{b}(x)=V_{0}$ and two examples of single-particle energies, representative of states that lie above and below the barrier. For the very thin barrier, described by the $\delta$-function potential, the odd states have a node at the barrier and so are not sensitive to it. Even states instead do not vanish at the barrier and their energy is increased by the interaction with the barrier.

To get an estimate for $V_{0}$ and $\Omega$, consider the characteristic energy of the discrete levels, caused by the perpendicular confinement:

$$
\epsilon_{0} \equiv \frac{\hbar^{2}}{2 m}\left(\frac{\pi}{x_{0}}\right)^{2}=0.38 \mathrm{eV}, \quad x_{0} \equiv 1.0 \mathrm{~nm} .
$$

Then one obtains that

$$
V_{0}=\epsilon_{0}\left(\frac{x_{0}}{\pi^{2} \varepsilon}\right)\left(x_{0} \Omega\right) .
$$

Taking $\varepsilon \sim 0.1 x_{0}$, then $V_{0} \sim 0.38 \Omega \mathrm{eV}$, where $\Omega$ is in $\mathrm{nm}^{-1}$.

In our calculations we use the physical parameters of niobium described in Tables I and II. We also list parameters for aluminum and lead, which are close and for which we expect similar results. All three materials reasonably satisfy the BCS theory relationship $2 \Delta / k_{\mathrm{B}} T_{\mathrm{c}} \approx 3.5$ [37-39]. Table I also contains other parameters needed for the study of the SIS nanofilm, such as $\Delta_{\text {bulk }}, E_{\mathrm{F}}^{3 \mathrm{D}}, e_{\mathrm{D}}$, and the Fermi wave number, $k_{\mathrm{F}}$. The chemical potential at zero temperature is fixed

TABLE I. The aluminum, lead, and niobium parameters required for the study of the SIS nanofilm. The critical temperature is given for completeness since our study is restricted to $T=0$. The bulk gap $\Delta_{\text {bulk }}$ is retrieved in the limit of a very thick film from Eq. (15). The bulk Fermi surface, $E_{\mathrm{F}}^{3 \mathrm{D}}$, sets the chemical potential for $T=0$, as given by Eq. (4). The Fermi wavelength $k_{\mathrm{F}}$ enters in Eq. (15), as shown in Ref. [17]. The Debye energy $e_{\mathrm{D}}$ is defined in Eq. (6).

\begin{tabular}{lccccc}
\hline \hline Metal & $T_{\mathrm{c}}(\mathrm{K})$ & $\Delta_{\text {bulk }}(\mathrm{K})$ & $E_{\mathrm{F}}^{3 \mathrm{D}}\left(10^{4} \mathrm{~K}\right)$ & $e_{\mathrm{D}}(\mathrm{K})$ & $k_{\mathrm{F}}\left(\mathrm{nm}^{-1}\right)$ \\
\hline $\mathrm{Al}$ & 1.2 & 1.97 & 13.6 & 433 & 17.5 \\
$\mathrm{Nb}$ & 9.3 & 17.7 & 6.18 & 276 & 11.8 \\
$\mathrm{~Pb}$ & 7.2 & 15.8 & 11.0 & 105 & 15.8 \\
\hline \hline
\end{tabular}


TABLE II. The aluminum, lead, and niobium energy parameters required for the study of the SIS nanofilm are shown here in units of the Debye energy, $e_{\mathrm{D}}=\hbar \omega_{\mathrm{D}}$. The coupling $\lambda$ is set to obtain the bulk gap $\Delta_{\text {bulk }}$ from Eq. (15) in the limit of a very thick film. The critical barrier strength $\Omega_{\mathrm{c}}$ is when cross pairing becomes important, as discussed in Sec. III.

\begin{tabular}{lccccc}
\hline \hline Metal & $\mu / e_{\mathrm{D}}$ & $\epsilon_{0} / e_{\mathrm{D}}$ & $\Delta_{\text {bulk }} / e_{\mathrm{D}}$ & $\lambda$ & $\Omega_{\mathrm{c}}\left(\mathrm{nm}^{-1}\right)$ \\
\hline $\mathrm{Al}$ & 314.1 & 10.08 & $4.56 \times 10^{-3}$ & 0.1640 & $2.21 \times 10^{3}$ \\
$\mathrm{Nb}$ & 223.9 & 15.81 & $6.41 \times 10^{-2}$ & 0.2901 & $2.46 \times 10^{2}$ \\
$\mathrm{~Pb}$ & 1048 & 41.56 & $1.51 \times 10^{-1}$ & 0.2889 & $2.75 \times 10^{2}$ \\
\hline \hline
\end{tabular}

and equal to the three-dimensional Fermi energy of the bulk system,

$$
\mu \equiv E_{\mathrm{F}}^{3 \mathrm{D}},
$$

and the multiple two-dimensional Fermi surfaces are defined by

$$
\epsilon_{\mathrm{F} j}^{2 \mathrm{D}}=\mu-\epsilon_{j}>0,
$$

where the discrete energy levels $\epsilon_{j}$ are discussed below. The Debye energy is defined as

$$
e_{\mathrm{D}} \equiv \hbar \omega_{\mathrm{D}}
$$

Table II shows these parameters in units of the Debye energy. It also shows the critical strength of the barrier, defined as $\Omega_{c}$, which is reached when the splitting between consecutive even and odd levels becomes comparable to the bulk gap, so that cross pairing must be considered. This is discussed in Sec. III. For $a=x_{0}$ [see Eq. (2)] the Debye energy is much smaller than the typical quantized energy across the film, $\epsilon_{0} / e_{\mathrm{D}}=15.81$. The chemical potential is significantly larger than the Debye energy $\mu \gg e_{\mathrm{D}}$, which justifies the analysis done in Sec. III. The dimensionless parameter $\lambda$, defined in Eq. (17), gives the strength of the pairing interaction and is determined from the requirement that the nanofilm gaps approach the bulk gap in the limit of increasing thickness.

We briefly summarize some key properties of the even and odd energy levels. Even and odd single-energy levels alternate in sequence, as shown in Figs. 3 and 4. The perpendicular discrete levels remain parabolic for any $\Omega$,

$$
\epsilon_{j}=\frac{1}{2 m} \hbar^{2} k_{j}(\Omega)^{2} .
$$

The odd levels are not affected by the barrier and their wave numbers are independent of $\Omega$ :

$$
k_{j}=j \pi / a, \quad j=1,2,3, \ldots
$$

The even levels have their wave numbers satisfying the condition

$$
k a \cot k a=-\Omega a,
$$

where, for a given $\Omega$, there are several possible solutions $k_{j}(\Omega)$. Useful analytic approximated solutions in the limit of a weak and strong potential barrier are obtained as follows: For $\Omega \sim 0$ we expand the solution as $k a=k_{0} a+\eta$, where $k_{0} a=(j+1 / 2) \pi$ is the solution for $\Omega=0$. Assuming $\eta$ small
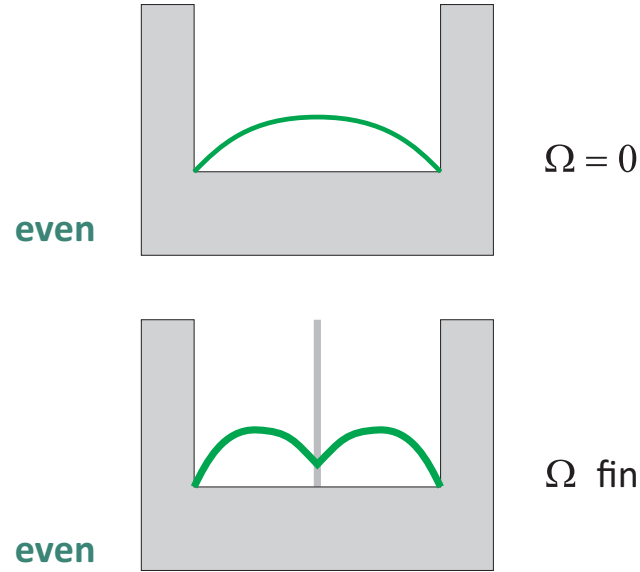

\section{$\Omega$ finite}

even

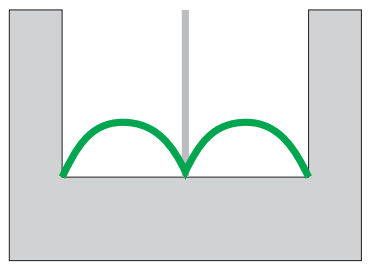

$\Omega=\infty$

even

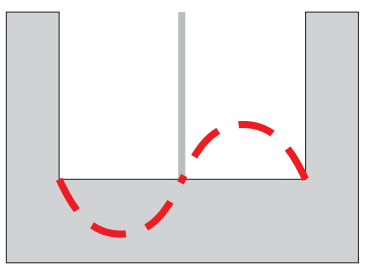

$\Omega \geq 0$

odd

FIG. 3. The wave function of the even single-particle ground state is represented as a function of the barrier strength $\Omega$, defined in Eq. (1). The first-excited state is odd and its wave function remains unchanged for any value of $\Omega$. The even and odd wave functions are shown in full (green) and dashed (red) lines, respectively.

we obtain

$$
k_{j} a \approx\left(j+\frac{1}{2}\right)+\sqrt{\left(j+\frac{1}{2}\right)^{2}+\Omega a} .
$$

We use the same strategy for $\Omega a \sim+\infty$ : given the solution $k_{0} a=j \pi$ for $\Omega=+\infty$, and assuming again $k a=k_{0} a+\eta$ with $\eta$ is small, we get

$$
k_{j} a \approx j \pi \frac{\Omega a}{1+\Omega a} .
$$

The physical interpretation of $\Omega$ becomes clear at this point. For $\left|k_{j}\right| \gg \Omega$, Eq. (9) reduces to $\cos k_{j} a \approx 0$ with the solutions for a nanofilm with no barrier inside $k_{j} \approx(2 j+1) \pi / a, j=$ $0,1,2 \ldots$

We now discuss how varying $\Omega$ changes the physics of the system. For $\Omega=0$ we have the Thompson-Blatt nanofilm [17] with no barrier inside. But we also have it in the limit $\Omega=$ $+\infty$ because two separated nanofilms each of width $a$ must be recovered by using the present formalism. The interesting physics appears for intermediate values of $\Omega$. Figure 3 shows how the even ground state changes according to $\Omega$, while the first-excited state, which is odd, is unchanged. Moreover, 


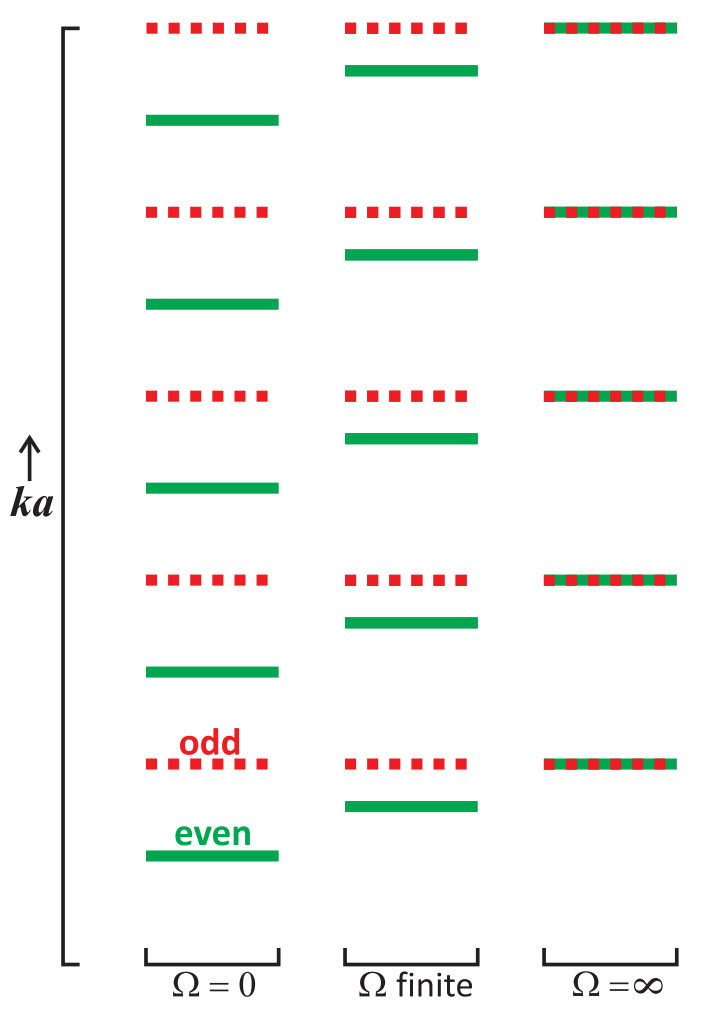

FIG. 4. Scheme of the single-particle energy levels according to their wave number represented by $k a$. Single-particle even and odd states are shown as filled (green) and dashed (red) lines, respectively. Three situations are displayed according to the potential strength defined in Eq. (1). For $\Omega=+\infty$, even and odd levels are degenerate.

in the limit $\Omega \rightarrow+\infty$ the ground state and the first-excited state approach the same state, modulo a phase, in each of the width $a$ nanofilms. This can be seen at the level of wave numbers in Fig. 4, for different values of $\Omega$. From $\Omega=0$ to $\Omega \rightarrow \infty$ even levels increase their energy and approach the odd ones. Figure 5 describes the multiple Fermi surfaces in the nanofilm. The first panel shows some of the parabolic bands and their intersection with the Debye energy window $\left(\mu-e_{\mathrm{D}}, \mu+e_{\mathrm{D}}\right)$. The second panel shows the result of the intersection of the parabolic bands with the chemical potential.
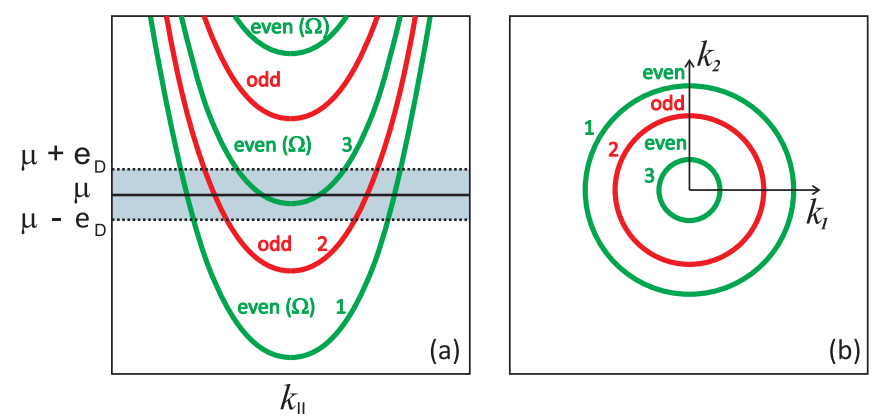

FIG. 5. Panel (a) shows the intersection between the parabolic bands, defined by Eq. (A17) and the Debye energy window, defined by Eq. (6). Panel (b) shows the multiple two-dimensional Fermi levels defined by Eq. (5). Even and odd states alternate in growing energy and are shown in distinct colors (green and red, respectively).
Even and odd Fermi surfaces succeed in sequence of increasing $j$ until a maximum $j_{\max }$ is reached beyond which $\epsilon_{\mathrm{F} j}^{2 \mathrm{D}}$ is no longer positive. Pairing occurs only within the Debye energy window around each of these two-dimensional Fermi surfaces.

\section{MULTIBAND AND MULTIGAP STRUCTURE OF SUPERCONDUCTOR-INSULATOR-SUPERCONDUCTOR NANOFILMS}

The SIS nanofilm can be regarded as a tunable system to study the interplay between parity and superconductivity because the insulating barrier lifts the degeneracy of even and odd single-particle states. While both the SIS and the single-slab nanofilms are multiband systems, the SIS nanofilm is always multigapped. The single-slab nanofilm is only multigapped within the shape-resonance region.

We assume a constant Debye energy window for all discrete levels, as in the Thompson-Blatt model [17]. The matrix elements are therefore given by

$$
V_{n m}=V_{i j} \theta\left(e_{\mathrm{D}}-\left|\zeta_{\vec{k}_{\|}, i}\right|\right) \theta\left(e_{\mathrm{D}}-\left|\zeta_{\vec{k}_{\|}^{\prime}, j}\right|\right),
$$

where $\theta$ is the Heaviside function, $\theta(x)=1$ for $x>0$ and $\theta(x)=0$ for $x<0$, and where we refer the reader to the appendix for the definition of the main quantities. The superconducting gap is homogeneous within each Debye energy window,

$$
\Delta_{n} \equiv \Delta_{j}\left(\vec{k}_{\|}\right)=\Delta_{j} \theta\left(e_{\mathrm{D}}-\left|\zeta_{\vec{k}_{\|}, j}\right|\right) .
$$

There is a maximum parallel energy $\epsilon_{\|}^{\max }=E_{2 j}=(\mu+$ $\left.e_{\mathrm{D}}-\epsilon_{j}\right) \theta\left[\mu+e_{\mathrm{D}}-\epsilon_{j}\right]$ and a minimum one, $\epsilon_{\|}^{\min }=E_{1 j}=$ $\left(\mu-e_{\mathrm{D}}-\epsilon_{j}\right) \theta\left[\mu-e_{\mathrm{D}}-\epsilon_{j}\right]$, which defines the parallel energy window, $\delta E_{\|} \equiv E_{2 j}-E_{1 j}$. In this paper we make the assumption that $\mu \gg e_{\mathrm{D}}$, so that the Debye and the (shape) resonance windows are small. We also take that the parallel electronic density of states is constant and given by the standard definition,

$$
N_{2 \mathrm{D}} / A=\frac{m}{2 \pi \hbar^{2}}=\frac{\pi}{4 x_{0}^{2} \epsilon_{0}} .
$$

Therefore, in the summation over single-particle states, we can substitute $\sum_{n}$ with $N_{2 \mathrm{D}} \int_{E_{1 j}}^{E_{2 j}} d \epsilon_{\|} \sum_{i}(\ldots)$. The equation that determines the superconducting gaps is obtained by integrating over the parallel momentum:

$$
\begin{aligned}
\Delta_{i}= & \frac{\pi \lambda}{k_{\mathrm{F}} 2 a} \sum_{j} V_{i j} F\left(\Delta_{j}\right) \Delta_{j}, \text { where } \\
F\left(\Delta_{j}\right)= & \frac{1}{2}\left\{\sinh ^{-1}\left[\frac{E_{2 j}+\epsilon_{j}-\mu}{\Delta_{j}}\right]\right. \\
& \left.-\sinh ^{-1}\left[\frac{E_{1 j}+\epsilon_{j}-\mu}{\Delta_{j}}\right]\right\},
\end{aligned}
$$

where the interaction matrix has been defined in the appendix.

The coupling $V$, introduced in the appendix, is related to the parameter $\lambda$ appearing in Table II via

$$
\lambda=\frac{k_{\mathrm{F}} N_{2 \mathrm{D}} V}{\pi A},
$$

where $k_{\mathrm{F}}$ is the three-dimensional Fermi wave number. 

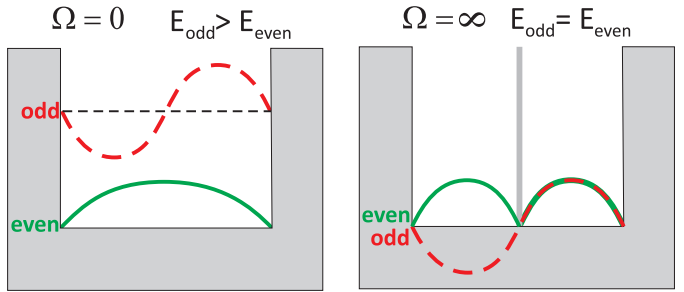

FIG. 6. The SIS nanofilm is represented here for the cases of no barrier $(\Omega=0)$ and of an infinite barrier $(\Omega=\infty)$ which are equivalent to a single and to two decoupled slabs, respectively. For $\Omega=0$ the single-particle ground state is even and the first-excited state is odd, whereas for $\Omega=\infty$ the single-particle even and odd states are degenerate in energy. The even and odd wave functions are shown in full (green) and dashed (red) lines, respectively.

It is instructive to briefly describe the limits $\Omega=0$ and $\Omega=\infty$ in order to make contact with the existing results of Thompson and Blatt [17]. In Fig. 6 the single-particle ground state and the first-excited one, which are respectively even and odd, are displayed for $\Omega=0$. For the single slab, the interaction matrix becomes $V_{i j}=1+\delta_{i j} / 2$. The singleparticle energies can be found in the appendix for convenience. Away from a shape resonance window, Eq. (15) becomes

$$
\Delta_{i}=\frac{\pi \lambda}{k_{\mathrm{F}} 2 a} \sum_{j=1}^{j_{\max }}\left(1+\frac{1}{2} \delta_{i j}\right) \sinh ^{-1}\left(\frac{e_{\mathrm{D}}}{\Delta_{j}}\right) \Delta_{j} .
$$

The maximum quantum level is $j_{\max }=$ floor $\left(\sqrt{\frac{\mu}{\epsilon_{0}}} \frac{2 a}{x_{0}}\right)$, where "floor" stands for the lowest integer and defines the number of activated bands. One can solve Eq. (18) assuming all superconducting gaps are equal, $\Delta_{i}=\Delta$, and find

$$
\frac{\Delta}{e_{\mathrm{D}}}=\sinh ^{-1}\left[\frac{2 k_{\mathrm{F}} a}{\pi \lambda\left(j_{\max }+\frac{1}{2}\right)}\right],
$$

which is the solution of Thompson and Blatt's original work [17]. We also confirm that all superconducting gaps are equal away from resonance from our numerical solution of Eq. (15), which is solved iteratively. A similar analysis is done for $\Omega=+\infty$, where one expects to recover two separated single-slab nanofilms.

Figure 6 shows the even and odd single-particle wave functions in the limit $\Omega=+\infty$. They have the same energy. Figure 7 shows the gap versus the half width $a$ in the limits $\Omega=$ 0 and $\Omega=+\infty$, as obtained from the single-gap solutions of Eq. (19) and its analog for $\Omega=+\infty$. To help visualize the equivalence between the $\Omega=0$ and $\Omega=+\infty$ limits, the points $a=1.063 \mathrm{~nm}$ and $a=2.126 \mathrm{~nm}$ are encircled. They yield the same gap value $\Delta / e_{\mathrm{D}}=7.05 \times 10^{-2}$.

As discussed in the appendix, for such an extreme limit $\Omega=$ $+\infty$, the wave functions coincide apart from a phase difference in each side of the barrier. The matrix elements between all possible combinations are found to be equal: $V_{i j}^{e, e^{\prime}}=V_{i j}^{o, o^{\prime}}=$ $V_{i j}^{e, o^{\prime}}=V_{i j}^{o, e^{\prime}}=1+\delta_{i j} / 2$. Thus one finds that, away from shape resonances, the gaps are all equal and given by Eq. (19) with the substitution $2 a \rightarrow a$ both in the argument of the hyperbolic sine and in the definition of $j_{\max }$.

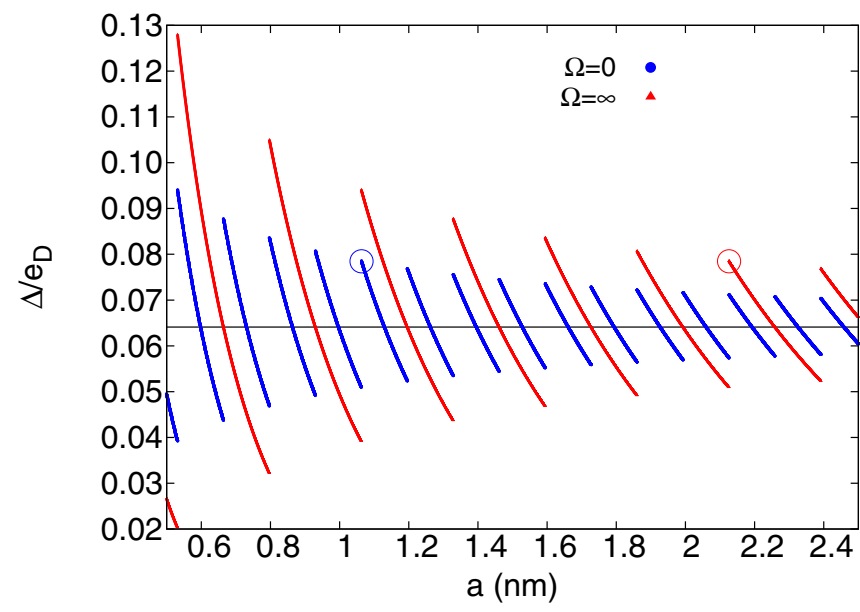

FIG. 7. The superconducting gap versus the half width $a$ within the window $0.5 \leqslant a(\mathrm{~nm}) \leqslant 2.5$, as obtained from Eqs. (19) and the analog for $\Omega=+\infty$. The two selected points marked by an asterisk represent the equivalence between these two curves. The $\Omega=0$ and the $\Omega=\infty$ curves are equivalent by a scaling $a \rightarrow 2 a$. The black horizontal line sets the value of the bulk gap. It only makes sense in this plot to consider widths $a_{K}=K a_{0}$; namely, those that are a multiple $K$ of the width of a single layer, $a_{0}\left(a_{0} \approx 0.1 \mathrm{~nm}\right)$.

Cross pairing must be considered in case the single-particle energy splitting becomes comparable to the bulk gap. This happens for a sufficiently strong barrier and the present $\mathrm{BdG}$ approach cannot be used anymore. Expanding the energy splitting between even and odd states using the exact value of the energy found in the appendix and Eq. (11), one obtains for a fixed $j$ and for $a \Omega$ large enough that

$$
\delta \epsilon_{j} \equiv \epsilon_{j}^{o}-\epsilon_{j}^{e} \sim j^{2}\left(\frac{\pi}{a}\right)^{2} \frac{\hbar^{2}}{2 m} \frac{1}{1+\Omega a} .
$$

A critical level $\Omega_{\mathrm{c}}$ is reached when the single-particle energy splitting becomes comparable to the bulk gap at $\delta \epsilon_{j}=\Delta_{\text {bulk }}$. As an example consider the case of a SIS nanofilm with total thickness $2.0 \mathrm{~nm}$; that is, $a=x_{0}$ [see Eq. (2)]. Since the smallest splitting is in the first level, $j=1$, one obtains that

$$
x_{0} \Omega_{\mathrm{c}}=\frac{\epsilon_{0}}{\Delta_{\text {bulk }}}-1,
$$

where $\epsilon_{0}$ is defined in Eq. (2). Recall that the discreteness along the perpendicular direction is based on $\epsilon_{0} \gg \Delta_{\text {bulk }}$.

\section{MULTIGAP FEATURES OF SUPERCONDUCTOR-INSULATOR-SUPERCONDUCTOR NANOFILM AT A SHAPE RESONANCE}

In this section, a Nb SIS nanofilm is studied within a very narrow half width window ( $a=1.060$ to $1.066 \mathrm{~nm}$ ) in the presence of a very weak insulating barrier $(a \Omega \ll 1)$. The emphasis here is to show that the SIS nanofilm (case $\Omega \neq 0$ ) is intrinsically multigapped for any width, while the single slab (case $\Omega=0$ ) is multigapped only inside the shape-resonance window, which is very narrow when the superconducting slab is made by conventional metals. Figure 8 shows the gaps in the case of no barrier $(\Omega=0)$, as obtained through Eqs. (19) 


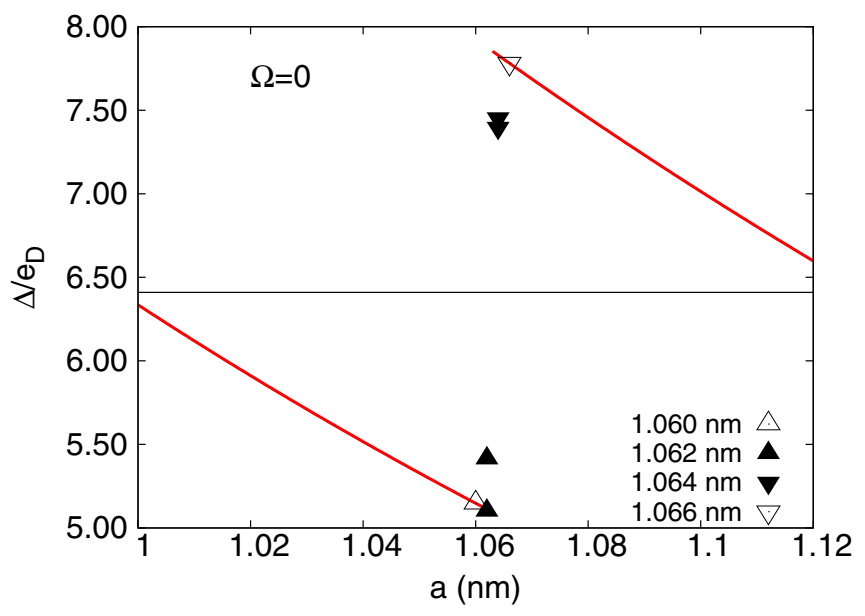

FIG. 8. The superconducting gaps in a shape resonance windows for $\Omega=0$. The (red) curve is obtained from Eq. (19), while the values for triangles are obtained from Eq. (18). At the extremities of the resonance window (white triangles) there is one superconducting gap, and a second gap appears inside the resonance window (black triangles). The black horizontal line sets the value of the bulk gap. The values $a=1.060$ and $1.066 \mathrm{~nm}$ are representative of any point outside the shape-resonance region. The gap is shown here a 100 times larger than its actual value.

and (15). The first equation gives the continuous (red) lines while the second one is used for specific points in the resonance region; namely, $a=1.060,1.062,1.064$, and $1.066 \mathrm{~nm}$. The points $a=1.060 \mathrm{~nm}$ and $a=1.066 \mathrm{~nm}$ are the extremities of the shape resonance. The entrance of a new odd band into the Debye energy window can also be seen in Table III. Recall that, according to Table II, $\mu / e_{\mathrm{D}}=223.9$.

We comment on the small variations that we consider for the parameter $a$, which are smaller than the size of the unit cell. There exist experimental techniques that can be used to achieve variations of the lattice of the order of 0.001 to $0.01 \mathrm{~nm}$. One is the electric field effect on metallic nanofilms, such as $\mathrm{Nb}$ composites, which moves the chemical potential

TABLE III. The single-particle energy levels in the case of no barrier and at the extremities of the shape-resonance region defined by $a$ and $a^{\prime}$, as described in Fig. 8. The number of energy levels changes from seven to eight, as shown here. The Debye energy window is $\mu / e_{\mathrm{D}}-1=222.9 . \mu / e_{\mathrm{D}}+1=224.9$, using the values of Table II. The Debye energy window lies below and above the highest energy level, for $a$ and $a^{\prime}$, respectively, thus leading to a shape resonance. Figures 8 and 9 show the onset of multigaps through this transition.

\begin{tabular}{lcc}
\hline \hline$\Omega=0$ & $a=1.060 \mathrm{~nm}$ & $a^{\prime}=1.066 \mathrm{~nm}$ \\
\hline Symmetry & $\epsilon_{j}(a) / e_{\mathrm{D}}$ & $\epsilon_{j}\left(a^{\prime}\right) / e_{\mathrm{D}}$ \\
\hline Even & 3.518 & 3.479 \\
Even & 31.66 & 31.31 \\
Even & 87.95 & 86.96 \\
Even & 172.4 & 170.5 \\
Odd & 14.07 & 13.91 \\
Odd & 56.29 & 55.68 \\
Odd & 126.7 & 125.2 \\
Odd & & 222.6 \\
\hline \hline
\end{tabular}

by about $10-100 \mu \mathrm{eV}[40,41]$, achieving the same effect on the chemical-potential position with respect to the electronic band structure as the one induced by the tiny variations of the SIS thickness considered in this work. Another is the pressure effect, applicable on nanofilms, which compresses the nanofilm by exactly the desired order of magnitude of 0.001 to $0.01 \mathrm{~nm}$ [42].

In Fig. 9 the $\Omega$ parameter is added. At the extremities of the shape-resonance region, while for $\Omega=0$ there is degeneracy between even and odd states, this is broken for $\Omega \neq 0$. The gap splitting due to the potential barrier is small $(\sim 0.5 \%)$ in comparison with the shape-resonance splitting $(\sim 3.0 \%)$. The even single-particle states in the range $0<a \Omega<1$, are

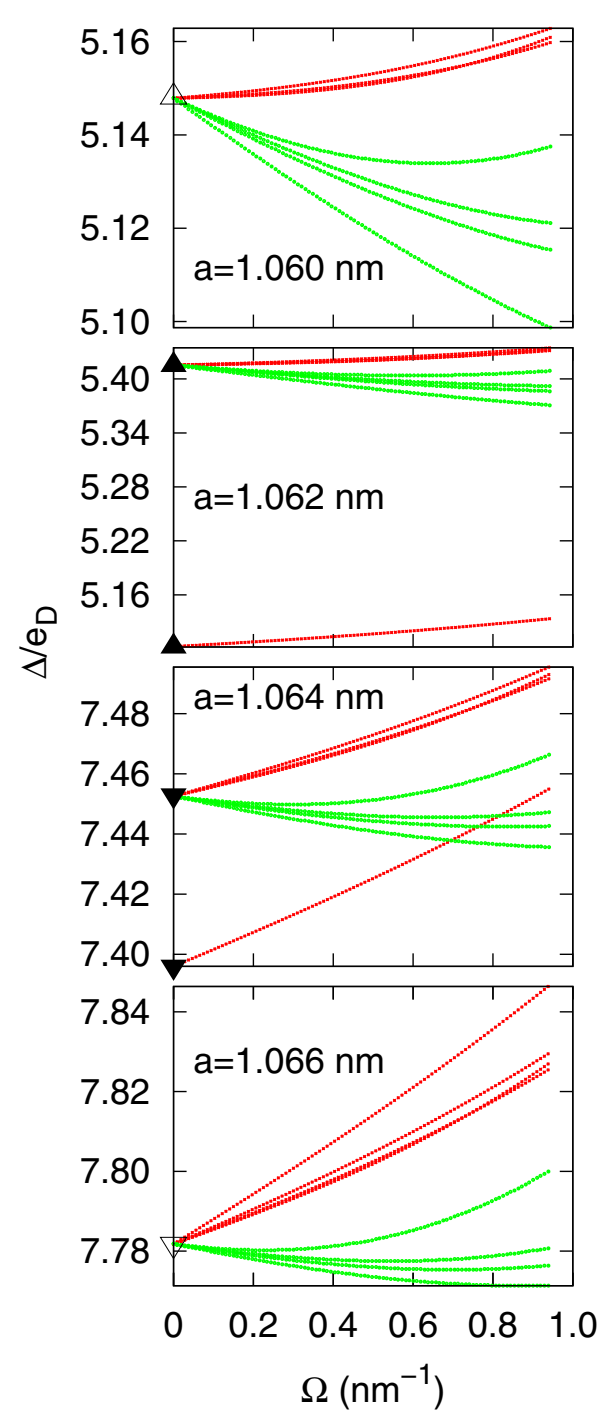

FIG. 9. The superconducting gaps of the SIS nanofilm for half widths $a=1.060,1.062,1.064$, and $1.066 \mathrm{~nm}$ versus the insulating barrier strength $\Omega$; see the text for a discussion on the tiny variation of the widths considered here. The $\Omega=0$ gaps, due to the shape resonance and also shown in Fig. 8, are marked by triangles. Even and odd gaps are presented by gray (green) and dark (red) lines, respectively. The insulating barrier $(\Omega \neq 0)$ lifts the degeneracy among the superconducting gaps. In particular, the multigap structure is a general feature outside the narrow shape resonance region. The gap is shown here a 100 times larger than its actual value. 


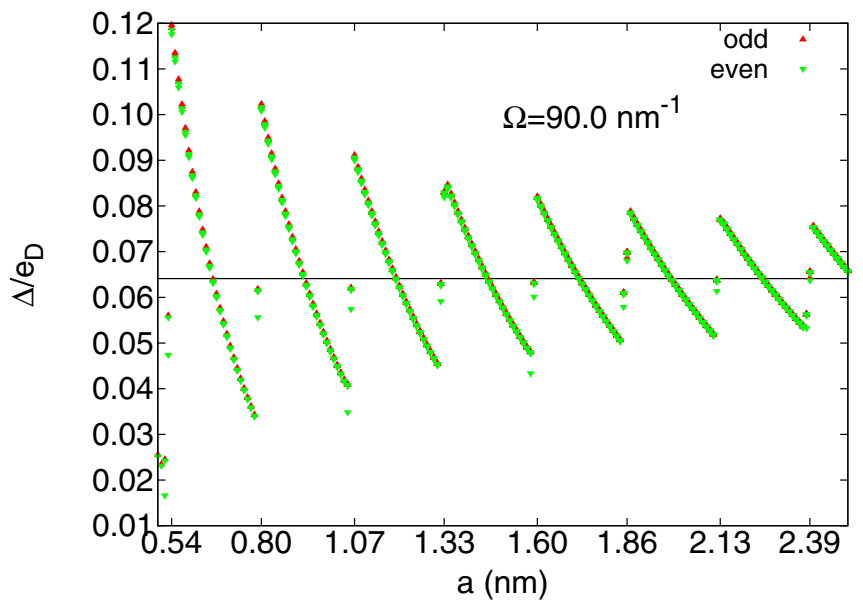

FIG. 10. The superconducting gap versus half thickness is shown within the window $0.5 \leqslant a(\mathrm{~nm}) \leqslant 2.5$. This is for a finite barrier whereas Fig. 7 corresponds to the extreme cases of no and of infinite barriers. This plot shows that shape resonances are also present in the case of a finite $(\Omega \neq 0)$ barrier. Even and odd gaps are presented by gray (green) and dark (red) lines, respectively. The tick marks on the $x$ axis are set at the top of the individual branches, where the onset of single-particle states is found with equal number of even and odd states. The first three branches can be found in Table IV. The black horizontal line sets the value of the bulk gap. Obviously it only makes sense in this plot to consider widths $a_{K}=K a_{0}$; namely, those that are a multiple $K$ of the width of a single layer, $a_{0}\left(a_{0} \approx 0.1 \mathrm{~nm}\right)$.

well described by Eq. (10). The gaps are obtained by solving Eqs. (15) and (16) with matrix elements given in Appendix B.

Figure 10 shows the gaps within the same half width window of Fig. $7,0.5 \leqslant a(\mathrm{~nm}) \leqslant 2.5$, now for a finite $\Omega=90 \mathrm{~nm}$, and Table IV has information about the number of

TABLE IV. This table contains the half widths where there are changes in the number of even and odd single-particle states, referred to by the pair of integers $(e, o)$. The barrier strength is $\Omega=90 \mathrm{~nm}^{-1}$. The number of even (odd) single-particle states is the same as the number of distinct even (odd) superconducting gaps whose maximum and minimum values are shown here. These gaps are also seen in Fig. 10. Notice that the highest superconducting gaps are always odd while the lowest ones are even.

\begin{tabular}{lccc}
\hline \hline & & $10^{2} \Delta_{e}^{\max } / e_{\mathrm{D}}$ & $10^{2} \Delta_{o}^{\max } / e_{\mathrm{D}}$ \\
$a(\mathrm{~nm})$ & $($ even, odd $)$ & $10^{2} \Delta_{e}^{\min } / e_{\mathrm{D}}$ & $10^{2} \Delta_{o}^{\min } / e_{\mathrm{D}}$ \\
\hline 0.50 & $(1,1)$ & 2.52 & 2.55 \\
0.52 & $(2,1)$ & 2.43 & 2.45 \\
0.52 & $(2,1)$ & 1.67 & 2.45 \\
0.54 & $(2,2)$ & 11.85 & 11.96 \\
0.54 & $(2,2)$ & 11.76 & 11.89 \\
0.79 & $(3,2)$ & 6.16 & 6.18 \\
0.79 & $(3,2)$ & 5.56 & 6.17 \\
0.80 & $(3,3)$ & 10.16 & 10.23 \\
0.80 & $(3,3)$ & 10.09 & 10.18 \\
1.05 & $(4,3)$ & 4.09 & 4.11 \\
1.05 & $(4,3)$ & 3.49 & 4.09 \\
1.07 & $(4,4)$ & 9.06 & 9.11 \\
1.07 & $(4,4)$ & 9.00 & 9.07 \\
\hline \hline
\end{tabular}

gaps and their range. The number of distinct gaps is the same as the number of accessible bands. Table IV shows the critical half widths where there is a change in the number of even and odd bands within part of the studied range $0.5 \leqslant a(\mathrm{~nm}) \leqslant 1.07$. We conclude from Fig. 10 that shape resonances still exist for finite $\Omega$. Nevertheless the shape resonance acquires a more elaborate structure since the number of even and odd single-particle levels, which here is the same as the number of distinct gaps, change in similar but not equal widths.

\section{INTERACTION-DRIVEN RESONANCE}

In this section we show that the adjustment of the barrier strength, $\Omega$, can bring the SIS nanofilm into resonance for a fixed half width $a$. The new phenomenon of barrier-driven resonance appears, which means that a sudden change of the superconducting gaps takes place without changing the half width $a$. We consider two Nb SIS nanofilms with fixed values $a=1.49 \mathrm{~nm}$ and $a=1.05 \mathrm{~nm}$. As discussed before, only even states are affected by the barrier, and so the cause of a barrierdriven resonance is the passage of an even single-particle state through the Debye energy window that makes a new even band accessible for pairing. Figures 11 and 12 show the gaps obtained by solving Eqs. (15) and (16).

The first example is for $a=1.49 \mathrm{~nm}$ and $a \Omega=1,2, \ldots 10$. The single-particle energy level $j=7$ lies above the Debye energy window and for this reason does not contribute to the superconducting state. The level $j=6$ falls below (or inside) the Debye energy window from $\Omega a=1.0$ to $\Omega a=6.0$, and above from 7.0 to 10.0. The single-particle energy level $j=5$ lies below the Debye energy window. Within this range of solutions the barrier-driven resonance can also be observed for other half widths in the proximity of $a=1.49 \mathrm{~nm}$. The barrierdriven resonance moves out of this fixed $a \Omega$ window for values of $a$ significantly different from that above. Figure 11 shows the gaps, as obtained from Eq. (15), versus the insulating barrier strength. An abrupt change of the gaps takes place near to $\Omega=4.03 \mathrm{~nm}^{-1}$ which characterizes the barrier-driven

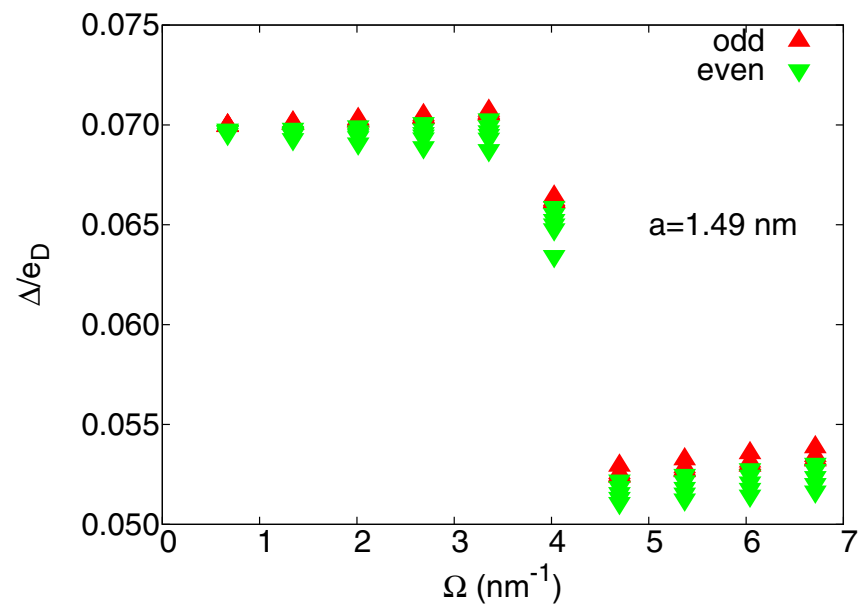

FIG. 11. A barrier-driven resonance is shown here for a fixed set of ten insulating barrier strengths, given by $a \Omega=1,2, \ldots 10$. Even and odd gaps are presented by gray (green) and dark (red) lines, respectively. The multigap structure of the SIS nanofilm is seen for a fixed $\Omega$. 


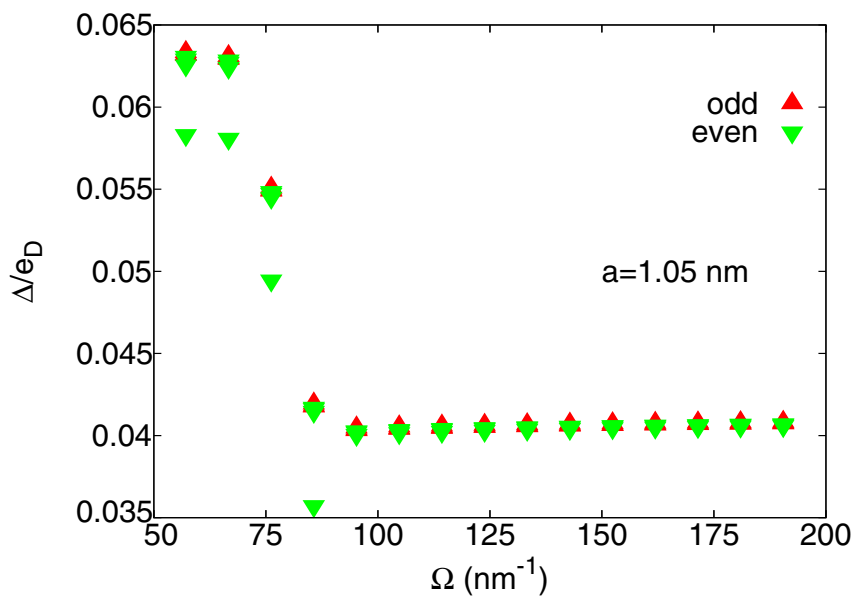

FIG. 12. A barrier-driven resonance is shown here within the range $50.0<a \Omega<200.0$ for a specific half width $a$. Notice that this range falls below the critical value $\Omega_{\mathrm{c}}$ of Eq. (21) and Table II. Even and odd gaps are presented by gray (green) and dark (red) lines, respectively. The distinct gaps are seen at fixed $\Omega$, showing the multigap structure of the SIS nanofilm.

resonance. Notice in this figure the multigap structure with odd gaps (triangles, red) above the even gaps (upside-down triangle, green). By increasing the barrier strength, the number of single-particle states inside and below the Debye energy window changes from 6 to 5 . The three values of $\Omega$ that characterize the transition are $\Omega=3.36$ (below), 4.03 (in), and 4.70 (above) $\mathrm{nm}^{-1}$. The odd single-particle energy levels are not affected by changes in the barrier strength. Figure 11 shows that the change in the gap values through the barrier-driven resonance can be quite high $(\sim 20 \%)$.

The second example is for the half width $a=1.05 \mathrm{~nm}$ and takes place in the range of potential barrier strength $50.0<$ $a \Omega<200$. In this example, the even single-particle states are obtained as approximate solutions of Eq. (9) given by Eq. (11). Figure 12 shows the multigaps versus $\Omega$ with significant changes in the gap at the barrier-driven resonance $(\sim 40 \%)$. The barrier-driven resonance observed at $\Omega=85.71 \mathrm{~nm}^{-1}$ also exist in a different $\Omega$ but still within the studied range for similar half widths. Notice that the studied range falls below $\Omega_{\mathrm{c}} \sim$ $246 \mathrm{~nm}^{-1}$ (Table II), beyond which cross-pairing between the even and odd levels must be included, which is not done here.

We conclude this section with a comment. The barrierdriven resonances share a common origin with the shape resonances, i.e., the entrance or exit of a single-particle state in or from the Debye energy window. In this respect they share the same behavior with respect to the main parameters that change them, which are the effective coupling and the width of the Debye window. An increase in the former leads to a more rounded seesaw pattern with smaller jumps, while an increase in the latter leads to a larger region in which the jump occurs. This behavior has been studied in detail for shape resonances in Ref. [43]. So the most pronounced resonances will happen for weakly coupled superconductors.

\section{DISCUSSION}

The study of nanostructured superconductors is important for many reasons, among them the quest to predict and realize metallic heterostructures that are multiband and multigap and present BCS-BEC crossover from momentum to position space pairing that can be tuned by system parameters. Theoretical and experimental evidence has shown that nanostructuring of bulk superconductors, in the form of nanofilms, nanostripes, and nanoclusters is able to induce multigap and multiband superconductivity and superconducting shape resonances $[16,18,44,45]$. Reference [46] contains an overview of the experimental state of the art for superconducting nanofilms. Multiband and multigap superconductors bring new paradigms to the understanding of coherent quantum phenomena, such as the enhancement of the superconducting critical temperature and the pairing energy gaps [47] through tunable parameters. The BCS-BEC crossover is also a central issue in present studies of high- $T_{\mathrm{c}}$ superconductivity. It has been proposed in aluminum-doped $\mathrm{MgB}_{2}$ [48] through a two-band, two-gap resonant superconductivity model. The BCS-BEC crossover has been studied in two-band and twogap superconductors [49] and also in quantum confined superconductors and superfluids [50,51]. Most important for connections with the results of the present work is the prediction that superconducting nanofilms can show BEC (molecule like) pairing induced by quantum confinement when the number of monolayer is reduced to a few units [52]. From the other side the shape resonances in superconducting gaps and critical temperature are expected to be generally present in a quasi-two-dimensional electron gas formed at an oxide-oxide interface [53], which is another system that could be used to realize the SIS structure investigated in this paper. Finally, we note that the shape resonances in superconducting nanofilms discussed at length in this paper are always accompanied by a topological change in the geometry of the Fermi surfaces, which is a Lifshitz transition. The Lifshitz transitions seem to be a general feature of high- $T_{\mathrm{c}}$ superconductors, in particular in iron-based systems. Key predictions associated with the Lifshitz transitions are resonances and amplifications in the superconducting critical temperature, as recently observed in electron-doped FeSe monolayers [54] and a typical chemicalpotential (density) dependence of the superconducting isotope effect on the critical temperature [55].

\section{CONCLUSION}

In this paper we have shown that a thin finite-height potential barrier between two identical superconducting slabs in the quantum size regime brings novel and interesting effects not found in single-superconducting-slab nanofilms. The even states are strongly affected by the insulating barrier whereas the odd states are not, and the pairing interaction between any two levels leads to a nontrivial splitting of the gaps. Consequently, both even and odd superconducting gaps feel the presence of the barrier. The SIS nanofilm is intrinsically multigapped, which means that it remains multigapped independently of any resonance condition while the single slab features multigaps only within the shape resonance region, which is generally narrow for metallic nanofilms. The multigap structure of the SIS nanofilm is homogeneous along the surface but not orthogonal to it because of quantum confinement [25]. The SIS nanofilm displays barrier-driven resonances which are distinct from the well-known Thompson-Blatt shape resonance. While 
barrier-driven resonances are controlled by the strength of the potential barrier in the middle, the shape resonances are controlled by the thickness of the superconducting slab. The results presented in this paper have been applied to a $\mathrm{Nb}-\mathrm{I}-\mathrm{Nb}$ nanofilm but the conclusions of intrinsic multigap superconductivity and barrier-driven resonances remain valid for other metals as well.

\section{ACKNOWLEDGMENTS}

We thank Antonio Bianconi, Mihaill Croitoru, and Pierbiagio Pieri for useful discussions. We also thank Natascia De Leo, Matteo Fretto, and Nicola Pinto for discussions on the possible experimental realizations of SIS nanofilms. Mauro M. Doria acknowledges $\mathrm{CNPq}$ support for funding (23079.014992/2015-39). Marco Cariglia acknowledges CNPq support for funding (205029/2014-0) and FAPEMIG APQ-02164-14. Andrea Perali acknowledges support from the University of Camerino FAR project CESEMN. The authors thank the colleagues involved in the MultiSuper International Network (http://www.multisuper.org) for the exchange of ideas and suggestions for this work.

\section{APPENDIX A: FORMALISM: THE ANDERSON APPROXIMATION TO THE BOGOLIUBOV -DE GENNES EQUATIONS FOR SUPERCONDUCTOR-INSULATOR- SUPERCONDUCTOR NANOFILM}

We briefly review the Anderson approximation [56] to the Bogoliubov-de Gennes equations [57] applied to the SIS nanofilm.

The energy of the excitations above the superconducting ground state at zero temperature, $E_{n}$, is obtained from the BdG equations:

$$
\left[\begin{array}{cc}
H_{0}(\mathbf{r}) & \Delta(\mathbf{r}) \\
\Delta^{*}(\mathbf{r}) & -H_{0}(\mathbf{r})
\end{array}\right]\left[\begin{array}{c}
u_{n}(\mathbf{r}) \\
v_{n}(\mathbf{r})
\end{array}\right]=E_{n}\left[\begin{array}{c}
u_{n}(\mathbf{r}) \\
v_{n}(\mathbf{r})
\end{array}\right],
$$

where the single-particle Hamiltonian is

$$
H_{0}(\mathbf{r})=-\frac{\hbar^{2}}{2 m} \nabla^{2}-\mu+U(\mathbf{r}) .
$$

The potential $U(\mathbf{r})$ confines particles inside the thin film and may contain an internal structure. The $\mathrm{BdG}$ equations are nonlinear in $u_{n}(\mathbf{r})$ and $v_{n}(\mathbf{r})$ since

$$
\Delta(\mathbf{r})=V \sum_{n} v_{n}^{*}(\mathbf{r}) u_{n}(\mathbf{r}),
$$

where $V$ is the strength of the pairing interaction $(V>0$ in the following). The number of particles and the density are given by

$$
N \equiv \int d \mathbf{r} n(\mathbf{r}), \quad n(\mathbf{r})=2 \sum_{n}\left|v_{n}(\mathbf{r})\right|^{2} .
$$

One seeks normalized wave functions:

$$
\int d \mathbf{r}\left[u_{n}^{*}(\mathbf{r}) u_{m}(\mathbf{r})+v_{n}^{*}(\mathbf{r}) v_{m}(\mathbf{r})\right]=\delta_{n, m} .
$$

In the case of a single superconducting slab, the summation index represents the set of parallel and perpendicular degrees of freedom, associated with continuous and discrete wave numbers, respectively.

In the Anderson approximation one solves the singleparticle Schrödinger equation

$$
H_{0}(\mathbf{r}) \Psi_{n}(\mathbf{r})=\zeta_{n} \Psi_{n}(\mathbf{r})
$$

and uses the following ansatz for the approximate solution to the $\mathrm{BdG}$ equations:

$$
u_{n}(\mathbf{r})=c_{n} \Psi_{n}(\mathbf{r}), \quad v_{n}(\mathbf{r})=d_{n} \Psi_{n}(\mathbf{r}),
$$

where the eigenfunctions are normalized:

$$
\int d \mathbf{r} \psi_{n}^{*}(\mathbf{r}) \Psi_{m}(\mathbf{r})=\delta_{n, m}
$$

A set of equations independent of position are then obtained as follows: The ansatz (A7) is introduced into Eq. (A1) and then multiplied by $\Psi_{n}^{*}(\mathbf{r})$. By using the normalization condition (A8) one obtains

$$
\left[\begin{array}{cc}
\zeta_{n} & \Delta_{n} \\
\Delta_{n} & -\zeta_{n}
\end{array}\right]\left[\begin{array}{l}
c_{n} \\
d_{n}
\end{array}\right]=E_{n}\left[\begin{array}{l}
c_{n} \\
d_{n}
\end{array}\right]
$$

The solution of the above equations is given by

$$
\begin{gathered}
c_{n}=\left[\frac{1}{2}\left(1+\frac{\zeta_{n}}{E_{n}}\right)\right]^{1 / 2}, \\
d_{n}=\left[\frac{1}{2}\left(1-\frac{\zeta_{n}}{E_{n}}\right)\right]^{1 / 2}, \text { and } \\
E_{n}=\sqrt{\zeta_{n}^{2}+\Delta_{n}^{2}},
\end{gathered}
$$

where

$$
\Delta_{n} \equiv \int d \mathbf{r} \Delta(\mathbf{r})\left|\Psi_{n}(\mathbf{r})\right|^{2} .
$$

Therefore the excitation energies $E_{n}$ are given in terms of the superconducting gaps $\Delta_{n}$ that must obey self-consistent equations, equivalent to Eq. (A3):

$$
\Delta_{n}=\sum_{m} V_{n m} \frac{\Delta_{m}}{2 \sqrt{\zeta_{m}^{2}+\Delta_{m}^{2}}} .
$$

The pairing interaction is given by the matrix elements:

$$
V_{n m}=V \int d \mathbf{r}\left|\Psi_{n}(\mathbf{r})\right|^{2}\left|\Psi_{m}(\mathbf{r})\right|^{2} .
$$

A detailed comparison between the Anderson approximation and the full BdG solution is reported in Ref. [58].

The BdG equations applied to the SIS nanofilm must take into account the perpendicular and parallel degrees of freedom of electron motion, such that the single-particle states, defined by Eq. (A6), satisfy

$$
\begin{gathered}
n \equiv\left(\vec{k}_{\|}, k_{j}\right), \\
\zeta_{n}=\epsilon_{\|}+\epsilon_{j}-\mu, \quad \epsilon_{\|}=\frac{\hbar^{2} \vec{k}_{\|}^{2}}{2 m}, \quad \epsilon_{j}=\frac{\hbar^{2} k_{j}^{2}}{2 m}, \\
\psi_{n}\left(\vec{x}_{\|}, x\right)=\frac{1}{\sqrt{A}} e^{i \vec{k}_{\|} \cdot \vec{x}} \psi_{j}(x) .
\end{gathered}
$$


The nanofilm surface area is $A$ and the wave-function component $\psi_{k_{j}}(x)$ satisfies the uni-dimensional Schrödinger equation,

$$
-\frac{\hbar^{2}}{2 m} \frac{d^{2} \psi_{k_{j}}}{d x^{2}}+U(x) \psi_{k_{j}}=\epsilon_{k_{j}} \psi_{k_{j}} .
$$

All $k_{j}$ states, $j$ being an integer, are bounded and for simplicity also labeled by $k$ or just by $j$. The wave functions are assumed to be normalized:

$$
\int_{-a}^{a} d x \psi_{j}^{*}(x) \psi_{j^{\prime}}(x)=\delta_{j j^{\prime}} .
$$

Thus, the general Eqs. (A6) and (A8) are reduced to the perpendicular ones, Eqs. (A19) and (A20), respectively. The dimensionless interaction matrix component in Eq. (A15) is associated with these wave functions defined below:

$$
V_{i j} \equiv 2 a \int_{-a}^{a} d x\left|\psi_{i}(x)\right|^{2}\left|\psi_{j}(x)\right|^{2},
$$

and $2 a$ is the total width of the SIS nanofilm.

\section{APPENDIX B: SOLUTION OF THE SCHRÖDINGER EQUATION}

Consider the one-dimensional Schrödinger equation of Eq. (A19) with the potential

$$
U(x)=\left\{\begin{array}{lll}
+\infty & \text { if } & |x| \geqslant a \\
\frac{\hbar^{2}}{m} \Omega \delta(x) & \text { if } & |x|<a .
\end{array}\right.
$$

The energy levels are given by $\epsilon_{k}=\frac{(\hbar k)^{2}}{2 m}$. There are two families of solutions according to their parity properties.

Even solutions are given by

$\psi_{k}(x)=\frac{1}{\sqrt{a-\frac{\sin 2 k a}{2 k}}}\left\{\begin{array}{rc}\sin k(x-a), & 0 \leqslant x \leqslant a \\ -\sin k(x+a), & -a \leqslant x \leqslant 0,\end{array}\right.$ where, according to Eq. (9), the wave number satisfies $k a \cot k a=-\Omega a$.

Odd solutions are given by

$$
\psi_{k}(x)=\frac{1}{\sqrt{a}}\left\{\begin{array}{l}
\sin k(x-a), \quad 0 \leqslant x \leqslant a \\
\sin k(x+a), \quad-a \leqslant x \leqslant 0,
\end{array}\right.
$$

where the wave number is given by $k a=j \pi, j=1,2,3, \ldots$.

The two limits of no barrier and infinite barrier represented in Fig. 6 are recovered as follows: In the limit of no barrier, $\Omega a=0$, the energy for even states is

$$
\epsilon_{j}=(2 j+1)^{2}\left(\frac{\pi}{2 a}\right)^{2} \frac{\hbar^{2}}{2 m},
$$

and that for odd states is

$$
\epsilon_{j}=j^{2}\left(\frac{\pi}{a}\right)^{2} \frac{\hbar^{2}}{2 m} .
$$

Instead, in the limit of an infinite barrier, $\Omega a=+\infty$, both the even states and odd states correspond to the energy levels,

$$
\epsilon_{j}=j^{2}\left(\frac{\pi}{a}\right)^{2} \frac{\hbar^{2}}{2 m} .
$$

As can be seen from the general formulas, the eigenstates are identical in each of the $-a \leqslant x \leqslant 0,0 \leqslant x \leqslant a$ intervals modulo a -1 factor.

For the potential considered in this section the generic matrix element (A21) can be expressed analytically as

$$
\begin{aligned}
V_{k k^{\prime}}= & \frac{1}{\sqrt{1-\frac{\sin 2 k a}{2 k a}}} \frac{1}{\sqrt{1-\frac{\sin 2 k^{\prime} a}{2 k^{\prime} a}}} \\
& \times\left\{1-\left[\frac{\sin 2 k a}{2 k a}+\frac{\sin 2 k^{\prime} a}{2 k^{\prime} a}\right]\right. \\
& \left.+\frac{1}{2}\left[\frac{\sin \left[2\left(k-k^{\prime}\right) a\right]}{2\left(k-k^{\prime}\right) a}+\frac{\sin \left[2\left(k+k^{\prime}\right) a\right]}{2\left(k+k^{\prime}\right) a}\right]\right\} .
\end{aligned}
$$

[1] A. Y. Liu, I. I. Mazin, and J. Kortus, Phys. Rev. Lett. 87, 087005 (2001).

[2] F. Giubileo, D. Roditchev, W. Sacks, R. Lamy, D. X. Thanh, J. Klein, S. Miraglia, D. Fruchart, J. Marcus, and P. Monod, Phys. Rev. Lett. 87, 177008 (2001).

[3] F. Giubileo, D. Roditchev, W. Sacks, R. Lamy, and J. Klein, Europhys. Lett. 58, 764 (2002).

[4] M. Iavarone, G. Karapetrov, A. E. Koshelev, W. K. Kwok, G. W. Crabtree, D. G. Hinks, W. N. Kang, E.-M. Choi, H. J. Kim, H.-J. Kim, and S. I. Lee, Phys. Rev. Lett. 89, 187002 (2002).

[5] M. Iavarone, R. Di Capua, A. E. Koshelev, W. K. Kwok, F. Chiarella, R. Vaglio, W. N. Kang, E. M. Choi, H. J. Kim, S. I. Lee, A. V. Pogrebnyakov, J. M. Redwing, and X. X. Xi, Phys. Rev. B 71, 214502 (2005).

[6] J. Kortus, I. I. Mazin, K. D. Belashchenko, V. P. Antropov, and L. L. Boyer, Phys. Rev. Lett. 86, 4656 (2001).

[7] A. Bianconi, M. Filippi, M. Fratini, E. Liarokapis, V. Palmisano, L. N. Saini, and L. Simonelli, Electron Correlation in New Materials and Nanosystems (Springer Netherlands, Dordrecht, 2007), Chap. II.1, pp. 93-101.
[8] H. Suhl, B. T. Matthias, and L. R. Walker, Phys. Rev. Lett. 3, 552 (1959).

[9] V. A. Moskalenko, M. E. Palistrant, and V. M. Vakalyuk, Sov. Phys. Usp. 34, 717 (1991).

[10] A. Perucchi, L. Baldassarre, S. Lupi, B. Joseph, and P. Dore, in Infrared, Millimeter, and Terahertz Waves (IRMMW-THz), 2012 37th International Conference (Wollongong, Australia, 2012), pp. 1-3.

[11] M. Abdel-Hafiez, Y.-Y. Zhang, Z.-Y. Cao, C.-G. Duan, G. Karapetrov, V. M. Pudalov, V. A. Vlasenko, A. V. Sadakov, D. A. Knyazev, T. A. Romanova, D. A. Chareev, O. S. Volkova, A. N. Vasiliev, and X.-J. Chen, Phys. Rev. B 91, 165109 (2015).

[12] A. Floris, A. Sanna, S. Massidda, and E. K. U. Gross, Phys. Rev. B 75, 054508 (2007).

[13] M. Ruby, B. W. Heinrich, J. I. Pascual, and K. J. Franke, Phys. Rev. Lett. 114, 157001 (2015).

[14] Y. Guo, Y.-F. Zhang, X.-Y. Bao, T.-Z. Han, Z. Tang, L.-X. Zhang, W.-G. Zhu, E. G. Wang, Q. Niu, Z. Q. Qiu, J.-F. Jia, Z.-X. Zhao, and Q.-K. Xue, Science 306, 1915 (2004). 
[15] X.-Y. Bao, Y.-F. Zhang, Y. Wang, J.-F. Jia, Q.-K. Xue, X. C. Xie, and Z.-X. Zhao, Phys. Rev. Lett. 95, 247005 (2005).

[16] T. Zhang, P. Cheng, W.-J. Li, Y.-J. Sun, G. Wang, X.-G. Zhu, K. He, L. Wang, X. Ma, X. Chen, Y. Wang, Y. Liu, H.-Q. Lin, J.-F. Jia, and Q.-K. Xue, Nat. Phys. 6, 104 (2010).

[17] C. Thompson and J. Blatt, Phys. Lett. 5, 6 (1963).

[18] A. Perali, A. Bianconi, A. Lanzara, and N. Saini, Solid State Commun. 100, 181 (1996).

[19] A. A. Shanenko and M. D. Croitoru, Phys. Rev. B 73, 012510 (2006).

[20] B. Josephson, Phys. Lett. 1, 251 (1962).

[21] A. A. Golubov, M. Y. Kupriyanov, and E. Il'ichev, Rev. Mod. Phys. 76, 411 (2004).

[22] J. Kim, V. Chua, G. A. Fiete, H. Nam, A. H. MacDonald, and C.-K. Shih, Nat. Phys. 8, 464 (2012).

[23] L. Serrier-Garcia, J. C. Cuevas, T. Cren, C. Brun, V. Cherkez, F. Debontridder, D. Fokin, F. S. Bergeret, and D. Roditchev, Phys. Rev. Lett. 110, 157003 (2013).

[24] L.-F. Zhang, L. Covaci, and F. M. Peeters, Europhys. Lett. 109, 17010 (2015).

[25] L.-F. Zhang, L. Covaci, and F. M. Peeters, Phys. Rev. B 91, 024508 (2015).

[26] K. Szałowski, Phys. Rev. B 74, 094501 (2006).

[27] D. Valentinis, D. van der Marel, and C. Berthod, Phys. Rev. B 94, 054516 (2016).

[28] D. Valentinis, D. van der Marel, and C. Berthod, Phys. Rev. B 94, 024511 (2016).

[29] A. M. García-García, J. D. Urbina, E. A. Yuzbashyan, K. Richter, and B. L. Altshuler, Phys. Rev. Lett. 100, 187001 (2008).

[30] S. Bose, A. M. García-García, M. M. Ugeda, J. D. Urbina, C. H. Michaelis, I. Brihuega, and K. Kern, Nat. Mater. 9, 550 (2010).

[31] J. Mayoh and A. M. García-García, Phys. Rev. B 90, 014509 (2014).

[32] J. Mayoh and A. M. García-García, Phys. Rev. B 90, 134513 (2014).

[33] D. Eom, S. Qin, M.-Y. Chou, and C. K. Shih, Phys. Rev. Lett. 96, 027005 (2006).

[34] S. Qin, J. Kim, Q. Niu, and C.-K. Shih, Science 324, 1314 (2009).

[35] R. Russo, C. Granata, A. Vettoliere, E. Esposito, M. Fretto, N. D. Leo, E. Enrico, and V. Lacquaniti, Supercond. Sci. Technol. 27, 044028 (2014).

[36] S. Flügge, Practical Quantum Mechanics, Classics in Mathematics (Springer-Verlag, Berlin, Heidelberg, 2013).

[37] C. Kittel, Introduction to Solid State Physics (John Wiley \& Sons, New York, 1976).
[38] N. Ashcroft and N. Mermin, Solid State Physics, Science: Physics (Saunders College, Philadelphia, 1976).

[39] J. W. Rohlf, Modern Physics from a to Z (Wiley, New York, 1994).

[40] M. Tortello, A. Sola, K. Sharda, F. Paolucci, J. R. Nair, C. Gerbaldi, D. Daghero, and R. S. Gonnelli, Appl. Surf. Sci. 269, 17 (2013).

[41] E. Piatti, D. Daghero, G. A. Ummarino, F. Laviano, J. R. Nair, R. Cristiano, A. Casaburi, C. Portesi, A. Sola, and R. S. Gonnelli, arXiv:1611.03132.

[42] E. Park, N. H. Lee, W. N. Kang, and T. Park, Appl. Phys. Lett. 101, 042601 (2012).

[43] M. Cariglia, A. Vargas-Paredes, M. M. Doria, A. Bianconi, M. V. Milošević, and A. Perali, J. Supercond. Nov. Magn. 29, 3081 (2016).

[44] A. Bianconi, A. Valletta, A. Perali, and N. Saini, Solid State Commun. 102, 369 (1997).

[45] A. Bianconi, A. Valletta, A. Perali, and N. L. Saini, Phys. C (Amsterdam, Neth.) 296, 269 (1998).

[46] A. A. Shanenko, J. A. Aguiar, A. Vagov, M. D. Croitoru, and M. V. Milošević, Supercond. Sci. Technol. 28, 054001 (2015).

[47] M. V. Milošević and A. Perali, Supercond. Sci. Technol. 28, 060201 (2015).

[48] D. Innocenti, N. Poccia, A. Ricci, A. Valletta, S. Caprara, A. Perali, and A. Bianconi, Phys. Rev. B 82, 184528 (2010).

[49] A. Guidini and A. Perali, Supercond. Sci. Technol. 27, 124002 (2014).

[50] A. Guidini, L. Flammia, M. V. Milošević, and A. Perali, J. Supercond. Novel Magn. 29, 711 (2016).

[51] A. A. Shanenko, M. D. Croitoru, A. V. Vagov, V. M. Axt, A. Perali, and F. M. Peeters, Phys. Rev. A 86, 033612 (2012).

[52] Y. Chen, A. A. Shanenko, A. Perali, and F. M. Peeters, J. Phys.: Condens. Matter 24, 185701 (2012).

[53] A. Bianconi, D. Innocenti, A. Valletta, and A. Perali, J. Phys.: Conf. Ser. 529, 012007 (2014).

[54] X. Shi, Z.-Q. Han, X.-L. Peng, R. Richard, T. Qian, X.-X. Wu, M.-W. Qiu, S. C. Wang, J. P. Hu, S. Y.-J., and H. Ding, arXiv:1606.01470.

[55] A. Perali, D. Innocenti, A. Valletta, and A. Bianconi, Supercond. Sci. Technol. 25, 124002 (2012).

[56] P. Anderson, J. Phys. Chem. Solids 11, 26 (1959).

[57] P. G. De Gennes, Superconductivity of Metals and Alloys, Advanced Book Classics (Perseus, Cambridge, 1999).

[58] K. Tanaka and F. Marsiglio, Phys. Rev. B 62, 5345 (2000). 